Mitja Ferenc*

Universität Ljubljana
UDK 323.15(497.434=112.2)(091)

DOI: $10.4312 /$ linguistica.60.2.227-243

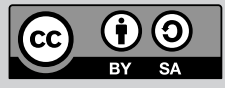

\title{
DAS SCHICKSAL DER DEUTSCHEN SPRACHMINDERHEIT IN SLOWENIEN
}

\section{DIE ANZAHL, ANWESENHEIT UND DIE POSITION DER DEUTSCHEN IN JUGOSLAWIEN UND IN SLOWENIEN BIS ZUM JAHR 1945}

Mit dem Zerfall der österreichisch-ungarischen Monarchie fanden sich die Deutschen in allen Staaten, die auf dem ehemaligen Gebiet der Monarchie neu entstanden - außer in Österreich - in der Lage einer ethnischen Minderheit wieder. Wie den Ergebnissen der im Jahr 1921 erfolgten Volkszählung zu entnehmen ist, lebten auf dem Gebiet des Königreichs der Serben, Kroaten und Slowenen („Königreich SHS“) damals 12 Millionen Menschen, davon stellten eine halbe Million bzw. rund $4 \%$ deutsche Muttersprachler dar. Sie formten die zahlenstärkste ethnische Minderheit, jedoch waren sie auf verschiedene Gebiete verstreut, ohne gemeinsame Tradition oder Organisationsverbände. Mit Ausnahme der Deutschen im slowenischen Raum gab es auch kein herausgebildetes nationales oder politisches Bewusstsein. Die Herkunft und soziale Zusammensetzung sowie die wirtschaftliche, kulturelle und politische Entwicklung der deutschsprachigen Minderheit war in einzelnen Gebieten des neuen Staates sehr unterschiedlich (Biber 1966: 11).

Zahlenmäßig am bedeutendsten war die Gruppe der jugoslawischen Deutschen (63 \%), die in der Provinz Vojvodina, d. h. in Banat, Bačka und Baranja lebte. Am kompaktesten waren ihre Ansiedlungen in Banat, wo sie gleich ein Fünftel der Gesamtbevölkerung darstellten. $25 \%$ jugoslawischer Deutschen lebten in Kroatien, Slawonien und in Srem, rund 4\% lebten in Bosnien und Herzegowina und $8 \%$ auf dem Gebiet des heutigen Sloweniens. (Biber 1966: 1-14).

Die Deutschen in Slowenien hatten eine reiche kulturelle und politische Tradition sowie ein stark ausgeprägtes Nationalbewusstsein. Sie waren in wirtschaftlicher und sozialer Hinsicht mit Abstand der stärkste und organisierteste Teil der deutschen Sprachminderheit. Sie nahmen eine wichtige Rolle in der Industrie, im Handel und in freien Berufen ein. Deswegen lag ihre Situation innerhalb der Gesellschaft weit über dem Durchschnitt der jugoslawischen Deutschen und auch weit über dem slowenischen Durschnitt, insbesondere in der Steiermark. Ein Viertel der Deutschen in Slowenien lebte vom Gewerbe, ein Fünftel vom Handel und etwas weniger als ein Fünftel von ihrer freiberuflichen Tätigkeit. $13 \%$ waren Arbeiter, etwas mehr als $5 \%$ öffentliche Beamte und Rentner und anderthalb Prozent waren Industrielle (Biber 1966: 27).

Die meisten Deutschen in Slowenien lebten in den Städten und Marktgemeinden der Steiermark (Maribor, Ptuj, Celje, Slovenske Konjice, Slovenska Bistrica usw.) und

Mitja.Ferenc@ff.uni-lj.si 
in Ljubljana, wo sie hauptsächlich Hausbesitzer, Industrielle, Freiberufler, Handwerker und Großgrundbesitzer, einigerorts aber auch Bauern waren. Im Süden Sloweniens lebten in einer kompakten, als deutsche Sprachinsel fungierenden Ansiedlung die Gottscheer Deutschen. Dorthin waren sie im 14. Jahrhundert von den Grafen von Ortenburg von deren Landgütern in Kärnten und im östlichen Tirol über- und angesiedelt worden.

Eine besondere Gruppe stellten in Slowenien diejenigen Deutschen dar, die durch den in Saint-Germain-en-Laye im Jahr 1919 abgeschlossenen Vertrag von dem mehr oder weniger kompakten deutschen Gebiet in Österreich abgeschnitten wurden. Dabei handelte es sich um die Deutschen im Apaško polje/Abstaller Feld, die durch die Grenze am Fluss Mura von der Obersteiermark getrennt wurden, und die Deutschen in vier Dörfern (Kramarovci, Ocinje, Fikšinci, Serdica) südlich vom Bach Kučnica im nordwestlichen Teil von Prekmurje/Übermurgebiet. Die Staatsgrenze wurde entlang dieses Baches gezogen (Zwitter 1938: 483-497).

Im Jahr 1910 lebten auf den Gebieten, die später ein Bestandteil des Königreichs SHS wurden, 106.377 Menschen mit Deutsch als Umgangssprache. Die deutschsprachige Bevölkerung stellte rund 10 \% der Gesamtbevölkerung dar, nach der Volkszählung in den Jahren 1921 und 1931 aber insgesamt etwas weniger als $4 \%$ bzw. 2,5 \%, als 28.998 Personen mit Deutsch als Muttersprache gezählt wurden (Ferenc, Repe 2004: 148-150, 162-165). Der Grund für eine solche statistische Verringerung der deutschen Bevölkerung ist die Uneinheitlichkeit der Volkszählung (Umgangssprache bzw. Muttersprache) und die Emigrationen, insbesondere des gebildeten Bürgertums und der Staatsbeamten, die aufgrund von staatlichen Maßnahmen ihre Lebensgrundlage verloren. Ebenso darf auch die Absicht slowenischer Behörden nicht vernachlässigt werden, deutschdenkende Einzelpersonen (die sog. „Deutschtümler ${ }^{\text {(e) }}$ zurück zum Slowenentum zu führen. Dabei muss berücksichtigt werden, dass sich bei der deutschen Agrarbevölkerung im Abstaller Feld und im Gottscheerland der bereits seit den Zeiten vor dem Ersten Weltkrieg herrschende Abwärtstrend fortsetzte. Andererseits war ein drastischer Rückgang der deutschsprachigen Bevölkerung in den Städten und Markgemeinden in der Steiermark sowie in Ljubljana festzustellen, wo die repressiven Maßnahmen gegen die deutsche Sprachminderheit am stärksten ausgeprägt waren. Diese zeigten sich nach dem Ende des Ersten Weltkriegs in der Auflösung der deutschen Privatschulen und in der Einschränkung des Unterrichts in deutscher Sprache in den Staatsschulen sowie durch die Auflösung der Minderheitsabteilungen und der Auflösung der meisten deutschen Vereine, in der Einschränkung der deutschen Sprache im öffentlichen Leben und der Einschränkung des Landgutbesitzes im Grenzgebiet. 
Tabelle 1: Die Anwesenheit der Deutschen in Slowenien während der Volkszählung in den Jahren 1910, 1921 und 1931

\begin{tabular}{|l|l|l|l|l|l|l|}
\hline & $\mathbf{1 9 1 0}$ & $\%$ & $\mathbf{1 9 2 1}$ & $\mathbf{\%}$ & $\mathbf{1 9 3 1}$ & $\mathbf{\%}$ \\
\hline $\begin{array}{l}\text { ŠTAJERSKA/ } \\
\text { STEIERMARK }\end{array}$ & $\mathbf{7 2 . 9 1 1}$ & 14,5 & $\mathbf{2 1 . 7 8 6}$ & 4,4 & $\mathbf{1 2 . 6 5 3}$ & $\mathbf{2 , 3}$ \\
\hline Celje/Cilli & 4625 & 66,8 & 859 & 11,1 & 449 & 5,9 \\
\hline Maribor/Marburg & 22.635 & 80,9 & 6.595 & 21,5 & 2.741 & 8,3 \\
\hline Ptuj/Pettau & 3672 & 79,3 & 968 & 21,8 & 559 & 13,1 \\
\hline Apaško polje/Abstaller Feld & 6000 & 65,0 & 4.847 & 52,5 & 2.921 & 30,2 \\
\hline KRANJSKA/KRAIN & $\mathbf{2 7 . 8 8 5}$ & $\mathbf{5 , 3}$ & $\mathbf{1 6 . 4 5 7}$ & $\mathbf{3 , 5}$ & $\mathbf{1 4 . 8 3 4}$ & $\mathbf{2 , 7}$ \\
\hline Ljubljana/Laibach & 6.742 & 14,5 & 1.826 & 3,4 & 1.729 & 2,9 \\
\hline $\begin{array}{l}\text { Gottscheerland/Kočevsko } \\
\text { (die Sprachinsel Gottschee) }\end{array}$ & 17.016 & 88,8 & 12.610 & 70,5 & 11.775 & 65,4 \\
\hline $\begin{array}{l}\text { KOROŠKA/KÄRNTEN } \\
\text { (Mießtal) }\end{array}$ & $\mathbf{3 . 0 7 6}$ & & $\begin{array}{l}\mathbf{7 4 5} \\
(\text { Bezirk }\end{array}$ & $\begin{array}{l}\mathbf{4 , 9} \\
\text { Prevalje) }\end{array}$ & $\begin{array}{l}\mathbf{1 . 0 3 7} \\
(\text { Bezirk }\end{array}$ & $\mathbf{3 , 2}$ \\
\hline $\begin{array}{l}\text { PREKMURJE/ } \\
\text { ÜBERMURGEBIET }\end{array}$ & $\mathbf{2 . 0 7 9}$ & & $\mathbf{2 . 5 4 0}$ & $\mathbf{2 , 8}$ & $\mathbf{1 . 4 8 9}$ & $\mathbf{1 , 6}$ \\
\hline \begin{tabular}{l} 
GESAMT \\
\hline
\end{tabular} & $\mathbf{1 0 6 . 3 7 7}$ & $\mathbf{9 , 4}$ & $\mathbf{4 1 . 5 1 4}$ & $\mathbf{3 , 9 3}$ & $\mathbf{2 8 . 9 9 8}$ & $\mathbf{2 , 5 3}$ \\
\hline
\end{tabular}

Die Pariser Friedenskonferenz sicherte den Deutschen im neuen jugoslawischen Staat einen minimalen, gesetzlich vorgesehenen Schutz für Minderheiten zu und auch das Königreich SHS musste einen besonderen Vertrag über den Schutz der ethnischen Minderheiten unterzeichnen, dessen Bestimmungen jedoch später in der VidovdanVerfassung nicht implementiert wurden. ${ }^{1}$ Auf diese Weise wollte das Königreich allen seinen Staatsbürgern eine Gleichstellung im Bereich der bürgerlichen und politischen Rechte, des Zugangs zur Beschäftigung und zu Berufen im öffentlichen Sektor sowie eine ungehinderte Nutzung der Minderheitssprache im öffentlichen Leben garantieren. Der Staat verpflichtete sich außerdem, auch für das öffentliche Minderheitsschulwesen in der Minderheitssprache Sorge zu tragen, allerdings nur überall dort, wo eine entsprechende Anzahl der Menschen lebte, die einer Sprachminderheit angehörten. Im nationalen Recht Jugoslawiens waren die Rechte ethnischer Minderheiten lediglich auf der Ebene von Einzelpersonen und nicht bezüglich Gruppen sichergestellt (Ferenc, Repe 2004: 150, 164, 165). Trotzdem wurden von den jugoslawischen/slowenischen

1 In der Vidovdan-Verfassung wurde nur im 16. Artikel Bezug auf die Minderheiten genommen, mit dem den »Minderheiten eines anderen Volksstammes und einer anderen Sprache« grundsätzlich das Recht auf das Schulwesen in der Muttersprache in Übereinstimmung mit dem gesetzlichen Bestimmungen gewährt wurde. In der oktroyierten Verfassung aus dem Jahr 1931 werden die ethnischen Minderheiten aber nicht erwähnt. 
Behörden in den ersten Jahren nach dem Umsturz keine Maßnahmen ergriffen, durch welche die Wirtschaftsmacht der deutschen Sprachminderheit geschwächt worden wäre. Somit wurde die Freiheit der Minderheitsangehörigen bei der Teilnahme am wirtschaftlichen Leben sichergestellt (Ferenc/Repe 2004: 154, 169, 170). ${ }^{2}$

Nach dem Umsturz im Jahr 1918 lösten die slowenischen Behörden die Niederlassungen des Deutschen Schulvereins und der Organisation Südmark auf und vorübergehend auch die meisten deutschen Vereine. Die zahlreichen Vereine erlebten in den ersten Jahren des neu entstandenen Staates ein sich wandelndes Schicksal. Die zentrale Kulturorganisation der Deutschen in Jugoslawien in der Zwischenkriegszeit oblag dem Schwäbisch-Deutschen Kulturbund (nachfolgend „Kulturbund“), der im Juni 1920 als ein nicht politischer Verband gegründet wurde, weil die Deutschen (bis zum Ablauf der mit den Friedensverträgen festgelegten Optionsfrist) kein Wahlrecht hatten. Daraufhin wurde der Kulturbund zum Mittelpunkt der Interessen der Deutschen, die sich früher an Aktivitäten der zahlreichen deutschen Gesangs-, Sport- und Feuerwehrvereine sowie der Kulturvereine (,„čitalnica“, dt. ,Lesesaal') und der Lesezirkel beteiligten. Der Kulturbund wurde mehrmals verboten und wiederhergestellt, jeweils in Abhängigkeit von den politischen Ausrichtungen, die die jeweiligen jugoslawischen Regierungen verfolgten. (Biber 1966: 33)

Zunächst erlaubten die Behörden in Slowenien im Jahr 1921 die Gründung der Gottscheer Bauernpartei (Kočevska kmečka stranka), in der sich die konservativ und katholisch orientierten Gottscheer Deutschen vereinigten. Im Jahr 1922 wurde in Maribor der Politische und wirtschaftliche Verein der Deutschen in Slowenien (Politično in gospodarsko društvo Nemcev v Sloveniji) gegründet. Die jugoslawischen Deutschen äußerten ihre Anforderungen und Wünsche meistens über die Partei der Deutschen des Königreiches der Serben, Kroaten und Slowenen (Stranka Nemcev Kraljevine Srbov, Hrvatov in Slovencev) (Biber 1966: 33, 35). Mit der Einführung der Diktatur im Jahr 1929 wurden alle Parteien, die auf nationalen Grundlagen basierten, aufgelöst. Die Deutschen in Slowenien gaben deswegen später die Anregung für die Gründung der Liga der Deutschen des Königreiches Jugoslawien für Völkerbund und Völkerverständigung (Nemška liga za Društvo narodov), die den Deutschen in Jugoslawien im Zeitraum der Diktatur als Ersatz für eine politische Organisation diente. Ihre Tätigkeit wurde im Jahr 1937 eingestellt (Biber 1966: 38).

Auch in der Zeit, als in den dreißiger Jahren des 20. Jahrhunderts die jugoslawischen Behörden freundschaftliche Beziehungen zum deutschen Staat unterhielten und die Angehörigen der deutschen Sprachminderheit einen besonderen Schutz genossen, schwand in Slowenien das deutsche Minderheitsschulwesen. Parallel zur Zahl der Schüler nahm auch die Zahl der deutschen Lehrer und Professoren ab. Im Schuljahr 1930/31 waren noch 36 Minderheitsschulen mit 54 Minderheitsabteilungen und 1.841 Schülern aktiv, nach 10 Jahren aber nur noch 14 Schulen mit 18 Abteilungen und 700 Schülern. Die Mehrheit befand sich im Gottscheerland (Kočevsko), weil die Dichte der deutschen Bevölkerung dort am höchsten war (Vovko 1986: 314-318).

2 In religiöser Hinsicht gehörten die slowenischen Deutschen überwiegend der römisch-katholischen Kirche an. Das Seniorat der deutschen evangelischen Gemeinden in Slowenien mit Sitz in Maribor zählte rund 2.100 Gläubige (Zajšek 2011: 100). 
Im Jahr 1939 wurde der Kulturbund von einer neuen Führung übernommen, die nationalsozialistisch orientiert war. Ihre Macht schöpfte sie aus den immer größeren Erfolgen des nationalsozialistschen Deutschland sowohl in der Wirtschaft als auch in der Politik, von dem sie über seine Organisationen erhebliche Unterstützung bekamen. Zuständig für die deutsche Sprachminderheit in Slowenien waren der Volksbund für das Deutschtum im Ausland und die Volksdeutsche Mittelstelle in Berlin. (T. Ferenc 1966: 98-130) Die Erfolge Deutschlands am Vorabend des Zweiten Weltkriegs weckten in den jugoslawischen Deutschen die Hoffnung, dass sie schon bald mit dem Deutschen Reich vereinigt werden würden. Diese Hoffnung wurde durch die deutschen Siege in Europa nur noch stärker die zunehmende Sympathie für den Nationalsozialismus wurde immer stärker deutlich.

\section{BESETZUNG}

Im April 1941 wurde das Gebiet des (heutigen) Slowenien, die Banschaft Dravska banovina, unter vier Staaten aufgeteilt. Deutschland besetzte den nördlichen Teil (Untersteiermark, Oberkrain, Mießtal), das Königreich Italien den südlichen Teil (Ljubljana, Unterkrain und Innerkrain) und Ungarn den nordöstlichen Teil (Prekmurje). Ein sehr kleiner Teil wurde dem neu entstandenen Staat Nezavisna država Hrvatska (NDH) zugeteilt. Italien und Ungarn schlossen das besetzte Land bald an ihre Staaten an, während das Deutsche Reich den Anschluss der zeitweiligen Verwaltungseinheiten Untersteiermark und „besetzte Gebiete von Kärnten und Krain“ hinausschob und letztendlich auf die Zeit nach dem Krieg verschob. Alle Besatzer führten harte systematische Assimilierungsmaßnahmen durch. Sie wandten Gewalt gegen die slowenische Bevölkerung an und wollten dadurch das slowenische Volk als ethnische Einheit verschwinden lassen. Die Mehrheit der deutschsprachigen Minderheit siedelte in dem von dem Deutschen Reich besetzten Gebieten. Als Gruppe organisiert, begrüßten sie die Besatzung und besetzten innerhalb der Besatzungsverwaltung die Stellen im politischen, administrativen und wirtschaftlichen Bereich. Zu Assimilierungszwecken wurden die Organisationen Štajerska domovinska zveza (dt. Steirischer Heimatbund) ${ }^{3}$ in der Steiermark und Koroška ljudska zveza (dt.: Kärntner Volksbund) ${ }^{4}$ in Oberkrain gegründet. In den beiden Organisationen wurde den ehemaligen Mitgliedern des Kulturbundes, die als Ganzes in diese Vereine aufgenommen worden und deutsche Staatsbürger geworden waren, eine besondere Rolle zugewiesen.

Die Deutschen im südlichen Teil waren angesichts der italienischen Besatzung enttäuscht. Deswegen siedelten sie sich in Übereinstimmung mit der Vereinbarung zwischen den beiden Staaten mehrheitlich in den vom Deutschen Reich besetzten Gebieten an. In nur wenigen Wochen bekamen rund 1.000 in Ljubljana lebende Deutsche und

3 Ein nationalsozialistischer Verein bzw. eine Organisation für die Assimilierung und Nazifizierung der Slowenen in der Untersteiermark unter der deutschen Besatzung Sloweniens zwischen den Jahren 1941-1945.

4 Nazistischer Verein bzw. eine Organisation, die während der deutschen Besatzung in der Zeit des Zweiten Weltkriegs für die Assimilierung und Nazifizierung auf den besetzten Gebieten des Kärntens und der Krain sorgte. 
rund 12.000 Gottscheer Deutsche eine „neue Heimat“ (Frensing 1970: 28). Die Gottscheer Deutschen wurden auf das Gebiet der Untersteiermark an den Flüssen Sava und Sotla, an der Grenze zu Italien und zum Unabhängigen Staat Kroatien (NDH), übersiedelt. Um diese Übersiedlung realisieren zu können, wiesen die nationalsozialistischen Behörden fast alle Slowenen aus diesen Gebieten, d. h. rund 37.000 Menschen, aus (T. Ferenc 1968: 587-592). Die Deutschen aus Ljubljana wurden in das Innere des deutschen Reiches umgesiedelt.

\section{AUSEINANDERSETZUNG MIT DEUTSCHEN}

Das Ende des Kriegs und die Niederlage Deutschlands im Jahr 1945 bedeutete auch das Ende der deutschen Sprachminderheit in Slowenien. Die Mitwirkung eines Teils dieser Minderheit bei der unmenschlichen Besatzungspolitik (massive Deportationen, Germanisierung, systematische Assimilierung, Gewalt) nutzten die jugoslawischen Nachkriegsbehörden zur endgültigen Abrechnung mit der deutschen Sprachminderheit im Staat (Repe 1998: 145).

Viele Deutsche verließen das slowenische Gebiet organisiert bereits vor dem Ende des Kriegs. Im Ausland wurden sie von den Alliiertenbehörden in Lagern interniert. Den Schätzungen zufolge verließen zwischen 15.000 und 16.000 Deutsche das Land noch vor dem Ende des Kriegs zusammen mit der sich zurückziehenden Armee (Repe 1998: 158). Wie der Rückzug und das Verlassen des Landes verliefen, können wir aus den schriftlich festgehaltenen Lebenserinnerungen erfahren (Dokumentation 1961; Lampeter/Kren 1994; T. Ferenc 1998: 120-123) und aus dem Archivbestand des Kulturbundes im Archiv der Republik Slowenien (Malnar 1997). Die neuen jugoslawischen/slowenischen Behörden beschlossen, jene Deutschen, die in Slowenien geblieben waren, des Landes zu verweisen.

Zunächst nahmen die Mitglieder der Abteilung für Volksschutz (OZNA) ${ }^{5}$, des Korps zur Volksverteidigung (KNOJ) ${ }^{6}$ und der Nationalmiliz, die Funktionäre der beiden Assimilierungsorganisationen sowie die Angehörigen des Kulturbundes fest. Im Juni und Juli 1945 wurde der Rest der auf dem Gebiet Sloweniens verbliebenen deutschen Bevölkerung verhaftet. Sie wurden in die für sie errichteten Lager gebracht. Das zentrale Konzentrationslager für die Deutschen aus dem ganzen Land hatte die OZNA Mitte Mai 1945 in Strnišče bei Ptuj (Sterntal), heute Kidričevo, errichtet. Weitere Internierungslager befanden sich in Hrastovec bei Sv. Lenart in Slovenske gorice, in Brestrnica bei Maribor, auf Studenci bei Maribor, in Kočevje und auf Teharje bei Celje (T. Ferenc 1998: 125, 135). In Slowenien wurden die Konzentrationslager bis Ende des Jahres 1945 aufgelöst, während in Jugoslawien die letzten Konzentrationslager für die Deutschen erst im Jahr 1948 aufgelöst wurden.

Die Ausweisung - Vertreibung der Deutschen erfolgte in drei Wellen. In der ersten

5 Abteilung für die nationale Sicherheit(OZNA), jugoslawische politische Polizei für die Bekämpfung des inneren Feindes sowie Durchführung aller Verfahren gegen die deutsche Sprachminderheit.

6 Das Korp der nationalen Verteidigung Jugoslawiens (KNOJ), ein besonderer Teil Jugoslawischer Armee für den Schutz der Außengrenzen und für die Bekämpfung des inneren Feindes. 
Welle, die von Mai bis September 1945 andauerte, wurden die aus dem Gottscheerland ausgesiedelten Deutschen verbannt. Bis Mitte des Sommers 1945 gab es auf dem Gebiet rund um die Flüsse Sava und Sotla keine deutschen Siedler mehr (M. Ferenc 2005: 271-275). Die zweite Welle, die bereits besser organisiert wurde, begann im September und dauerte bis November 1945, als OZNA und KNOJ die Emigrationen wegen der Wahlen in Jugoslawien vorübergehend unterbrachen. Diese Welle umfasste diejenigen, die in Konzentrationslagern interniert worden waren. Die Mehrheit wurde nach Österreich verbannt. Die letzte Phase der vollkommenen „Säuberung“ Sloweniens von der deutschsprachigen Volksgruppe fand von Ende Dezember 1945 bis Juni 1946 statt. Diese Phase umfasste fast alle Deutschen aus Slowenien (49 Transporte bzw. Gruppen). Die Verbannung erfolgte hauptsächlich über die jugoslawisch-österreichische Grenze. Als die in Österreich stationierten englischen Besatzungsbehörden die Vertriebenen nicht mehr annehmen wollten, begannen die jugoslawischen Behörden in Absprache mit den sowjetischen Besatzungsbehörden in Ungarn, die Deutschen über die ungarische Grenze zu verbannen. Von den Sowjets wurden die Vertriebenen weiter nach Österreich und zum Teil nach Deutschland transportiert (T. Ferenc 1998: 139). Gemäß den Daten der Geheimpolizei wurden 9.474 Personen ausgesiedelt. ${ }^{7}$ Zudem wurden mehrere Hundert Deutsche aus Slowenien in das Konzentrationslager Kničanin in der Provinz Vojvodina gebracht.

Um ihr Vermögen konfiszieren zu können, wurden mehrere Dutzend Deutsche vor den Militärgerichten verurteilt, viele auch in Abwesenheit. Es wird geschätzt, dass ungefähr ein Zehntel derjenigen Deutschen, die in Slowenien blieben, d. h. zwischen 1.000 und 1.500, liquidiert wurden oder infolge der schlechten Lebensbedingungen in den Konzentrationslagern starben. In Slowenien werden bereits seit einigen Jahren die Opfer des Zweiten Weltkriegs namentlich aufgenommen. Unter den 15.000 Personen, die nach dem Ende des Kriegs von den neuen kommunistischen Behörden hingerichtet worden waren, wurden 522 Deutsche aus Slowenien namentlich verzeichnet. Wie den Dokumentationen zu entnehmen ist, gab es aber noch 709 Personen mit überwiegend deutschen Familiennamen, die in den Sammellagern starben oder dort hingerichtet worden waren. Ihre Nationalität ist noch nicht zuverlässig nachgewiesen. ${ }^{8}$ In Strnišče wurden viele internierte Menschen bereits bei der Ankunft in das Lager ohne ein Gerichtsverfahren hingerichtet. Sie wurden in den nahegelegenen Schottengruben erschossen und eingegraben. Eine größere Anzahl der Gefangenen aus Strnišče und den Gefängnissen in Maribor wurde in das Pohorje-Gebirge gebracht und dort hingerichtet. Auch die Kranken wurden nicht verschont (Zavadlav 1990: 91-93). In den letzten Jahren wurden in Slowenien mehrere sogenannte verdeckte Grabstätten eingerichtet, in denen die Angehörigen der deutschen Minderheit hingerichtet werden sollten. Ein bitteres Schicksal erlebten auch die Kinder der hingerichteten Eltern, die aus

\footnotetext{
7 Archiv der Republik Slowenien (ARS), AS 1931, Schachtel 1062, Bericht über die Umsiedlungen der Volksdeutschen in den Jahren 1945-1946, 26.11.1951.

8 Opfer des Zweiten Weltkriegs: http://www.sistory.si/zrtve (01.11.2014). Schreiben des Instituts für Zeitgeschichte (Inštitut za novejšo zgodovino) an den Autor, 07.03.2012.
} 
den Konzentrationslagern in staatliche soziale Einrichtungen geschickt wurden. Die bekannteste dieser Einrichtungen ist das Kinderlager Pri Petričku in Celje.

Durch die Gesetzgebung wurde für die Deutschen die sogenannte Kollektivschuld geltend gemacht, woraufhin sie zu Feinden des Staates und des Volkes erklärt wurden (Repe 1998: 151-153). Unter den Vorschriften, die die Rechtslage der Deutschen in Jugoslawien nach der Befreiung regelten, war auch die erste und wichtigste Verordnung des Vorsitzes des Antifaschistischen Rates der Nationalbefreiung Jugoslawiens (AVNOJ) vom 21. November 1944. ${ }^{9}$ Mit dieser Verordnung wurde der Besitz des deutschen Staates, der deutschen Staatsbürger und der Personen deutscher Nationalität in Slowenien und Jugoslawien beschlagnahmt und in das Eigentum des Staates überführt.

\section{4 „DIE DEUTSCHEN“ IM NEUEN STAAT}

Durch die Vertreibung und die Konfiszierung ihres Besitzes verloren die Angehörigen der deutschen Sprachminderheit auch ihre jugoslawische Staatsbürgerschaft (Repe 1998: 154). Die Rechtssituation der Deutschen, die im Staat blieben oder aus den Konzentrationslagern zurückkehrten, besserte sich nach dem Jahr 1951, als für Deutschland, Österreich und ihre Staatsbürger die Vorschriften, die infolge der Kriegssituation mit Deutschland eingeführt worden waren, nicht länger Anwendung fanden (Nečak 1998: 228). Ähnliche Entscheidungen im Hinblick auf die Deutschen wurden auch von anderen Staaten getroffen. Rund 12 Millionen Angehörige der ethnisch deutschen Bevölkerungsgruppe wurden aus Polen, der Tschechoslowakei, Ungarn, Jugoslawien, Rumänien, Bulgarien, der Sowjetunion und den baltischen Ländern vertrieben (Nečak 2004: 362).

Nach der Vertreibung aus Slowenien/Jugoslawien und nach der zeitweiligen Unterbringung in den Konzentrationslagern in Kärnten und der Steiermark in Österreich zerstreuten sich die Angehörigen der deutschen Minderheit und wurden in allen Regionen Österreichs und Deutschlands sesshaft. Viele entschieden sich aber für den Weg nach Amerika. Die Auseinandersetzung mit den Deutschen in der Nachkriegszeit geschah so gründlich, dass in Slowenien nur noch weit verstreute „Reste von Resten“ der einmal starken Sprachenminderheit blieben. Die „Deutschen" ${ }^{10}$ in Jugoslawien blieben meistens ohne ihren Besitz und ohne Minderheitenschutz. Die Verbesserung ihrer Lage setzte nach 1948 ein, als sie die Staatsbürgerschaft beantragen konnten. Nach einem Jahr bekamen sie dann auch alle anderen bürgerlichen Ehrenrechte, einschließlich des Wahlrechtes. Für die jugoslawischen Behörden galt die „deutsche Frage“ zu diesem Zeitpunkt als endgültig geklärt. Das tragische Schicksal, das sie erlebten, war die Folge der historischen Ereignisse: der harten nazionalsozialistischen Besatzungspolitik, der Paranoia vor einer Wiederholung, der Euphorie ob des Sieges und der damit verbundenen revanchistischen Maßnahmen. Deswegen wollten die meisten in Jugoslawien

9 „Verordnung des AVNOJ-Präsidiums über den Übergang des Besitzes des Feindes in den Besitz des Staates, über die Verwaltung des Besitzes abwesender Personen durch den Staat und über die Konfiszierung des Besitzes, der von den Besatzungsbehörden zwangsweise alieniert wurde.“ Amtsblatt des Demokratischen Föderativen Jugoslawiens. 06.02.1945.

10 Die Bezeichnung „Deutsche“ meint die Personen mit ethnisch deutscher oder österreichischer Herkunft sowie die Personen mit Deutsch als Muttersprache. 
verbliebenen „Deutschen“ das Land trotz ihrer verbesserten Lage so schnell wie möglich verlassen.

Angesichts des Schicksals, das die deutsche Minderheit während des Kriegs und in der Nachkriegszeit hinnehmen musste, überrascht es nicht, dass die Anzahl der Deutschen und Österreicher (nach dem Kriterium der Nationalität) bzw. der deutschen Muttersprachler bei den Volkszählungen nach dem Krieg sehr niedrig war. Der Anteil dieser Menschen erreichte nicht einmal ein Zehntel derjenigen, die vor dem Krieg auf slowenischem Gebiet lebten. Obwohl die damaligen Volkszählungen keine eindeutige Wiedergabe des Standes und der Lage der „Deutschen“ bei uns darstellen, lassen sich folgende Charakteristika feststellen: Im Mittelpunkt steht eine zahlenmäßig kleine Gemeinschaft, die territorial verstreut und nicht autochthon ist und für die eine hohe Umzugsfrequenz und eine spezifische, vom slowenischen Durchschnitt abweichende Strukturierung der Bevölkerung charakteristisch sind.

Bei der Volkszählung im Jahr 1948 wurden 1.824 Deutsche und 582 Österreicher (insgesamt 2.406 Personen) gezählt. Bei dieser Volkszählung war ihre Anzahl am größten, danach sank die Zahl kontinuierlich. Die Volkszählung im Jahr 1953 ergab 1.906 „Deutsche“ und 2.509 Personen mit Deutsch als Muttersprache, im Jahr 1961 betrug die Anzahl von „Deutschen“ noch 986, zehn Jahre später 700 und im Jahr 1981 nur noch 560 „Deutsche“ und 1.189 Personen mit Deutsch als Muttersprache. Die Volkszählung im Jahr 1991 stellte 745 Personen fest, die ihre deutsche und österreichische Nationalität erklären, und 1.543 Personen, die angaben, dass Deutsch ihre Muttersprache sei. Neben der geringen Anzahl ist ein weiteres Merkmal der "Deutschen“ in Slowenien ihre Streuung, d. h. ihre Verteilung auf dem Gesamtgebiet Sloweniens. ${ }^{11}$ Gemäß der Volkszählung im Jahr 1991 lebten sie in 309 Siedlungen und die Personen mit Deutsch als Muttersprache in 459 Siedlungen. Nirgends wohnten Österreicher, Deutsche und deutsche Muttersprachler derart konzentriert in einem Ballungsraum. In keinem Ort und keiner Gemeinde erreichte ihre Konzentration ein Prozent der Gesamtbevölkerung. Auch innerhalb der einzelnen Ansiedlungen war ihre Anzahl im Verhältnis zur Mehrheitsbevölkerung klein.

Nach dem Kriterium des Autochthonismus kann die Mehrheit der Personen österreichischer und deutscher Nationalität nicht als Nachkommen der aus der Vorkriegszeit stammenden deutschen Minderheit auf dem Gebiet Sloweniens eingestuft werden. Gleich eine Hälfte der Personen, die ihre österreichische oder deutsche Nationalität erklärten, wanderte nämlich aus dem Ausland und $7 \%$ aus den ehemaligen Republiken Jugoslawiens ein. Mehr als die Hälfte wurde im Ausland geboren.

Ferner treten die Deutschen in Slowenien durch die für sie charakteristische, hohe Umzugsfrequenz (zeitweiliger Aufenthalt im Ausland) und auch durch die vom slowenischen Durchschnitt abweichende Struktur hervor. Männer stellen nur ein Drittel dar, die Kinderzahl ist gering, es handelt es sich um eine gealterte Population, der Anteil der vorübergehend im Ausland lebenden Personen ist hoch und die Familien sind im Hinblick auf die Nationalität nicht homogen (M. Ferenc 1998: 271-287).

11 Mehr als eine Hälfte der Personen beider Nationalitäten lebt in den Siedlungen mit einer bis drei Personen der einen oder der anderen Nationalität. 
Im Jahr 2002 wurde in Slowenien die letzte klassische Volkszählung durchgeführt. Es wurden nur 963 Personen mit Deutsch als Muttersprache und nach dem Kriterium der Nationalität 181 Österreicher und 499 Deutscher gezählt. Später wurden die Daten über die Nationalität und Religion nicht länger erfasst (M. Ferenc 2011: 33-37).

Unter den Gebieten, die durch die Aussiedlung und Verbannung der Deutschen gravierende Folgen erlitten, war auch das Gottscheerland (Kočevsko). Wahrscheinlich könnte europaweit nur mit Müh und Not ein Gebiet gefunden werden, in dem die Kulturlandschaft von vergleichbaren Veränderungen geprägt wurde. Die Aussiedlung von 12.000 Gottscheern Deutschen, Kriegsschädigung, die ungeeignete Ansiedlungs-, Personal-, Wirtschafts- und Kulturpolitik in der Nachkriegszeit sowie die systematische Zerstörung, insbesondere sakraler Objekte, aus nationalen und ideologischen Gründen - durch all diese Ereignisse und Faktoren wurde verursacht, dass große Teile des Gottscheerlandes (Kočevsko) mit Wäldern überwachsen, die Siedlungen zerstört und die Überreste verdeckt oder zerstört wurden (Ferenc 1993). Von den ehemaligen 176 Dörfern sind mehr als 100 nicht mehr vorhanden (M Ferenc 2018). Neben den vielen Siedlungen ist für das Gebiet des Gottscheerlandes (Kočevsko) besonders die Zerstörung der Sakralgebäude und -objekte als großer Verlust zu beklagen. Von 123 Kirchen wurden 95 zerstört. Ein ähnliches Schicksal erlebten auch Kapellen sowie Holz- und Steinmale. Von den ehemals mehr als 400 ist heute nur noch ein Zehntel begeh- bzw. sichtbar (Ferenc, 2007). Die meisten von den ehemaligen 38 Friedhöfen wurden aufgelöst, eingeebnet oder die Grabsteine wurden entfernt, die davon zeugten, dass in dieser Gegend einst die Deutschen lebten (Ferenc, 2002). Heute erinnern nur noch wenige materielle Überreste an die 600-jährige Anwesenheit der deutschen Sprachminderheit im Gottscheerland (Kočevsko).

\section{ORGANISATION DER DEUTSCHEN IN DER REPUBLIK SLOWENIEN}

Nach den demokratischen Veränderungen im Jahr 1990 wurden in Slowenien mehrere deutsche Vereine gegründet. Der älteste Verein ist die „Freiheitsbrücke“ (Društvo Most svobode), gegründet im Dezember 1990 in Maribor. Dieser Gründung folgten die Vereine, die mit der deutschen Sprachinsel im Gottscheerland (Kočevsko) verbunden sind: der „Gottscheer Altsiedler-Verein“ (Društvo Kočevarjev staroselcev) und der „Verein Peter Kosler“ (Slovensko kočevarsko društvo Peter Kosler). Der ersterwähnte Verein wurde im Jahr 1992 von den Gottscheer Deutschen (Kočevarji) aus dem Tal Črmošnjiško-Poljanska dolina gegründet. Die Mehrheit der Mitglieder stellen die Gottscheer Deutschen (Kočevarji) aus dem ehemaligen Bezirk von Novo mesto dar, sowohl diejenigen, die im Jahr 1941 nicht umzogen, als auch ihre Nachkommen und Sympathisanten. Der slowenische Gottscheer Verein Peter Kosler aus Ljubljana wurde im Jahr 1994 gegründet und war bis 2018 aktiv. Benannt wurde dieser Verein nach Peter Kosler, dem Kartografen aus dem Gottscheerland (Kočevsko), der auch Autor der ersten Landkarte slowenischer Länder ist. ${ }^{12} \mathrm{Im}$ Jahr 2000 wurde auf Initiative der in Maribor und Umgebung lebenden deutschsprachigen Bevölkerung in Slowenien der „Kulturverein deutschsprachiger Frauen ,Brücken““ (Kulturno društvo

12 Verein Peter Kosler http://www.gottscheer.eu/kosler/index.html (01.12.2018). 
nemško govorečih žena Mostovi) gegründet. ${ }^{13}$ In Apače ist seit dem Jahr 2007 der Kulturverband Kulturno društvo Josef Matl aktiv. Der jüngste Verein in Slowenien ist aber der Kulturverein Cilli an der Sann (Kulturno društvo Celje ob Savinji). Gegründet wurde der Verein im Jahr 2011 auf Initiative der in Celje und Umgebung lebenden deutschsprachigen Bevölkerung. ${ }^{14}$ Der „Kulturverein deutschsprachiger Jugend“ (Kulturno društvo nemško govoreče mladine) wurde von den jungen Nachkommen der Deutschen aus der Steiermark und Krain gegründet. ${ }^{15}$ Abschließend gilt es noch den Dachverband der Gottscheer Organisationen (Zveza kočevarskih organizacij), gegründet im März 2018, zu erwähnen. ${ }^{16}$

In den Programmen all dieser Vereine ist die Sorge für die Erhaltung des Kulturerbes der Deutschen im slowenischen Raum festegelegt, insbesondere für die Erhaltung sprachlicher, ethnischer und kultureller Charakteristika der deutschsprachigen Bevölkerung Sloweniens. Einige davon haben sich auch die verfassungsrechtliche, mit den autochthonen Minderheiten - der italienischen und der ungarischen - gleichgestellten Anerkennung der deutschen Sprachminderheit in Slowenien zum Ziel gesetzt. Die Tätigkeit der Vereine ist durch Finanz- und Personalprobleme geprägt. Sie erwarten vonseiten des slowenischen Staates eine größere Unterstützung als die, die ihnen auf der Grundlage der Kulturvereinbarung mit der Republik Österreich zugewiesen wird.

\section{DIE FRAGE DER ANERKENNUNG DER DEUTSCHEN SPRACHMINDERHEIT IN DER REPUBLIK SLOWENIEN}

Die Republik Österreich sprach im Jahr 1992, bald nach der Anerkennung der Republik Slowenien, das Thema der Anerkennung der deutschen Volksgruppe in Slowenien an. Diese Frage wurde zu einer Konstanten in den zwischenstaatlichen Beziehungen zwischen Slowenien und Österreich (Jančar 1996). Viele Jahre lang widersprach Slowenien der Erwähnung der Deutschen in Slowenien als Minderheit. Gesprächen über diese Frage wurde von Slowenien u. a. auch wegen der Befürchtung zugestimmt, dass Österreich Slowenien am EU-Beitritt hindern könnte. Das Thema der deutschen Sprachminderheit wurde in der Öffentlichkeit immer stärker mit den Anforderungen an die Auflösung der „AVNOJ-Beschlüsse“ und auch mit den Denationalisierungsanforderungen der österreichischen Staatsbürger verbunden. Zwar verabschiedete Slowenien im Jahr 1991 das Gesetz über die „Denationalisierung“. Jedoch hat sich Slowenien im Zuge dessen verpflichtet, das konfiszierte Vermögen nur denjenigen Personen zurückzugeben, die in der Zeit der Konfiszierung die jugoslawische Staatsbürgerschaft innehatten. ${ }^{17}$ Die jugoslawische Staatsbürgerschaft hatten die über- und ausgesiedelten Deutschen allerdings nicht besessen. Das Verfassungsgericht in Slowenien annulierte den Grundsatz der kollektiven Schuld, der in der Verordnung des AVNOJ-Präsidiums

13 http://www.drustvo-mostovi.si/ (01.12.2018).

$14 \mathrm{http}: / / w w w . a l t o e s t e r r e i c h e r . n e t / k u l t u r n o-d r u s t v o-c e l j e-o b-s a v i n j i /(01.12 .2018)$.

$15 \mathrm{http} / / / w w w . a l t o e s t e r r e i c h e r . n e t /(01.12 .2018)$.

16 Dachverband der Gottscheer Organisationen http://www.gottscheer.eu/deutsch.xhtml (01.12.2018).

17 Gesetz über die Denationalisierung, Amtsblatt der RS, Nr. 27/91, vom 29.11.1991. 
aus dem Jahr 1944 festgelegt wurde, zum Teil. Das Verfassungsgericht gab den Angehörigen der deutschen Sprachminderheit die Möglichkeit, nachzuweisen, dass sie während der Besatzung nicht illoyal waren. Wenn sie dies belegen konnten, hatten sie die Möglichkeit, sowohl die slowenische Staatsbürgerschaft zu erhalten als auch ihr Vermögen zurückzuerhalten. Österreich beurteilte diese Erklärung des Gerichts als diskriminatorisch (Ferenc/Repe 2004: 642-645, 693-697).

Dieses politisch sehr sensible Thema versuchten die Staaten mit Hilfe von Experten zu lösen. So entstanden auf slowenischer wie auch auf österreichischer Seite wissenschaftliche Studien über die deutschsprachige Bevölkerung der Republik Slowenien (Nečak 1998a, 1998b; Karner 1998). Gegründet wurde zudem ein slowenisch-österreichischer Ausschuss, der die Beziehungen zwischen den beiden Staaten im 20. Jahrhundert analysierte. Der slowenische Teil der Kommission veröffentlichte seine Ergebnisse im Jahr 2004 (Nečak et al.: 2004).

Die Staaten lösten das Problem der verfassungsrechtlichen Anerkennung der deutschen Volksgruppe in Slowenien durch den Abschluss eines Kulturabkommens im Jahr 2001. ${ }^{18}$ In diesem Abkommen ist festgelegt, dass die Rechte der Angehörigen der deutschsprachigen Volksgruppe im Artikel 61 der Verfassung der Republik Slowenien ${ }^{19}$ sichergestellt sind und nicht in Artikel 64, der die Lage der autochthonen ungarischen und italienischen Minderheit regelt. Dadurch sollten die Trennlinien zwischen den individuellen und kollektiven Rechten deutlich genug gezogen werden. In der slowenischen Verfassung steht demnach geschrieben, dass auf dem Gebiet der Republik Slowenien neben Slowenen zwei autochthone ethnische Gemeinschaften leben, und zwar die italienische und die ungarische Minderheit. Neben diesen zwei Minderheiten wird in der Verfassung darüber hinaus die Roma-Gemeinschaft explizit erwähnt, der durch die Verfassung ebenfalls ein besonderer Status eingeräumt wird. Der deutschsprachigen Gemeinschaft wird der Status einer autochthonen ethnischen Gemeinschaft von unserem Staat nicht zuerkannt, genauso wenig wie den Kroaten, Serben, Bosniern, Mazedoniern, Albanern und noch einigen anderen Volksgruppen, die in Slowenien leben. In Slowenien wurde der Unterzeichnung des Abkommens eher zurückhaltend zugestimmt, während diese Tat für die österreichische Seite den ersten Schritt zur Anerkennung ihrer Minderheit in Slowenien bedeutete.

Es ist jedoch eine Tatsache, dass das Kulturabkommen weder die deutschsprachige Gemeinschaft in Slowenien noch die Republik Osterreich zufriedenstellt, die eine verfassungsrechtliche Anerkennung der deutschen Sprachminderheit in Slowenien und Rechte erwarten, die mit denen der italienischen und ungarischen Minderheiten vergleichbar wären. Im Mai 2018 nahm das österreichische Parlament einstimmig

18 Abkommen zwischen der Regierung der Republik Slowenien und der Regierung der Republik Österreich über die Zusammenarbeit auf dem Gebiet der Kultur, Ausbildung und Wissenschaft, Amtsblatt der RS - Internationale Verträge, Nr. 5/02. Von österreichischer Seite wurde das Abkommen im Juli 2001 offiziell bestätigt.

19 Artikel 61: „Jeder Mensch hat das Recht, seine Zugehörigkeit zu seiner Nation oder ethnischen Gemeinschaft frei zu erklären, seine Kultur zu pflegen und zu äußern sowie seine Sprache und seine Schrift zu verwenden“; Verfassung der RS, Amtsblatt Nr. 33/9, 23.12.1991. 
die Resolution zur Unterstützung der offiziellen Anerkennung der deutschsprachigen Gemeinschaft in Slowenien als Minderheit an. Das Außenministerium wurde mit der Weiterverfolgung der Bemühungen um die offizielle Anerkennung als Minderheit beauftragt. ${ }^{20}$ Slowenien vertritt weiterhin die Position, dass die Verfassung nicht geändert werden solle ${ }^{21}$ und dass das Kulturabkommen aus dem Jahr 2001 einen geeigneten Rahmen für die Aktivitäten und Projekte der Angehörigen der deutschsprachigen Volksgruppe in Slowenien biete. ${ }^{22}$

\section{Literatur}

Archiv der Republik Slowenien (ARS) AS 1931, Schachtel 1062, Bericht über die Umsiedlungen der Volksdeutschen in den Jahren 1945-1946, 26.11.1951.

BIBER, Dušan (1966) Nacizem in Nemci v Jugoslaviji 1933-1941. Ljubljana: Cankarjeva založba.

Bundesministerium f. Vertriebene (1961) Dokumentation der Vertreibung der Deutschen aus Ost-Mitteleuropa. Das Schichsal der Deutschen in Jugoslawien. Bd. 5. Düsseldorf: BMV.

FERENC, Mitja (1993) Kočevska. Izgubljena kulturna dediščina kočevskih Nemcev / Gottschee. Das verlorene Kulturerbe der Gottscheer Deutschen. Ljubljana: Zavod RS za varstvo naravne in kulturne dediščine.

FERENC, Mitja (1998) ,„Nemci“ na Slovenskem v popisih prebivalstva po drugi svetovni vojni.“"In: D. Nečak (Hrsg.), 271-322.

FERENC, Mitja (2005) Kočevska pusta in prazna. Nekdanje nemško jezikovno območje na Kočevskem po odselitvi Nemcev. Ljubljana: Modrijan.

FERENC, Mitja (2007) Nekdanji nemški jezikovni otok na Kočevskem / Die ehemalige Sprachinsel im Gottscheerland / Former German linguistic island in Kočevje region. Kočevje: Pokrajinski muzej Kočevje.

FERENC, Mitja (2011) „Etno- in socialnodemografska struktura »Nemcev« na Slovenskem v obdobju jugoslovanske države po 2. svetovni vojni.“ In: J. Malačič/M. Gams (Hrsg.) Soočanje z demografskimi izzivi, Zbornik 14. mednarodne multikonference Informacijska družbe. Heft B, 33-37.

FERENC, Mitja/Joachim HÖSLER (2011) Spurensuche in der Gottschee: deutschsprachige Siedler in Slowenien. Potsdam: Deutsches Kulturforum östliches Europa.

20 Wien: Unterstützung der Anerkennung der deutschsprachigen Gemeinschaft in Slowenien als Sprachminderheit https://www.rtvslo.si/svet/dunaj-podpora-priznanju-nemsko-govoreceskupnosti-v-sloveniji-kot-manjsine/455273 (01.12.2018).

21 Der damalige Außenminister Erjavec antwortete der Republik Österreich: „Slowenien sieht keine Änderungen auf dem Gebiet der ethnischen Minderheiten vor.“ https://www.rtvslo.si/ slovenija/erjavec-odgovarja-avstriji-slovenija-sprememb-na-podrocju-narodnih-manjsin-nenacrtuje/455360 (01.12.2018).

22 Sobotka für die Änderung des Status der deutschsprachigen Volksgruppe in Slowenien, Pahor ist dagegen https://www.rtvslo.si/slovenija/sobotka-za-spremembo-statusa-nemsko-govoreceskupine-v-sloveniji-pahor-proti/481912 (01.12.2018). 
FERENC, Mitja/Božo REPE (2004) „Nemška manjšina v Sloveniji med obema vojnama / Die deutsche Minderheit in Slowenien in der Zwischenkriegszeit". In: D. Nečak (et al.) (Hrsg.), 147-176.

FERENC, Mitja/Gojko ZUPAN (2006) Cerkve na Kočevskem nekoč in danes II. Opisi cerkva nekdanjega nemškega jezikovnega območja v župnijah Črmošnjice, Črnomelj, Planina, Semič/Die Kirchen im Gottscheerland einst und heute II. Beschreibungen der Kirchen der ehemaligen deitsvhen Sprachinsel in den Pfarren Tschermoschnitz. Črnomelj, Stockendorf, Pöllandl und Semič. Ljubljana: ZRC SAZU.

FERENC, Mitja/Gojko ZUPAN (2018) Gottschee Villages in Slovenia. The Gottschee Germans Used to Live among Us. Part I: A-J. Ljubljana: Znanstveni inštitut Filozofske fakultete.

FERENC, Mitja/Gojko ZUPAN/Mateja BAVDAŽ (2002) Pokopališča in nagrobniki kočevskih Nemcev / Friedhöfe und Grabsteine der Gottscheer Deutschen / Cemeteries and tombstones of the Gootscheer Germans. Ljubljana: Zavod za varstvo kulturne dediščine Slovenije.

FERENC, Tone (1968) Nacistična raznarodovalna politika v Sloveniji v letih 19411945. Maribor: Obzorja, 587-592.

FERENC, Tone (1998) „Nemci na Slovenskem med drugo svetovno vojno“. In: D. Nečak (Hrsg.), 99-144.

FRENSING, Hans Hermann (1970) Die Umsiedlung der Gottscheer Deutschen. Das Ende einer südostdeutschen Volksgruppe. München: Verlag R. Oldenbourg.

JANČAR, Matjaž (1996) Slovenska zunanjepolitična razpotja. Beograd: Međunarodna politika.

KARNER, Stefan Karner (1998) Die deutschsprachige Volksgruppe in Slowenien. Aspekte ihrer Entwicklung 1939-1997. Klagenfurt/Ljubljana/Wien: Hermagoras Verlag.

LAMPETER, Wilhelm/Ludwig KREN (Hrsg.) (1987) 35 Jahre Gottscheer Landsmannschaft in Deutschland. Weilheim: Gottscheer Landsmannschaft Deutschland.

LAMPETER, Wilhelm/Ludwig KREN (Hrsg.) (1994) Gottscheer Fluchtlingsschicksale. Berichte über Umsiedlung und Vertreibung sowie die Zeit danach. Weilheim: Gottscheer Landsmannschaft Deutschland.

MALNAR, Mateja (1997) Analiza fonda Kulturbunda v Arhivu MNZ. Diplomska na$\log a$. Univerza v Ljubljani. Ljubljana: Oddelek za zgodovino Filozofske fakultete.

NEČAK, Dušan (1998) „'Nemci“ na Slovenskem 1945-1955 v luči nemških in avstrijskih dokumentov." In: D. Nečak (Hrsg.), 173-244.

NEČAK, Dušan (Hrsg.) (1998a) „Nemci“ na Slovenskem 1941-1955. Izsledki projekta. Ljubljana: Znanstveni inštitut Filozofske fakultete.

NEČAK, Dušan (Hrsg.) (1998b) Die „Deutschen“ in Slowenien (1918-1955). Kurzer Abriss/»Nemci« na Slovenskem (1918-1955). Kratek oris. Ljubljana: Znanstveni inštitut Filozofske fakultete.

NEČAK, Dušan (et al.) (Hrsg.) (2004) Slovensko-avstrijski odnosi v 20. stoletju/Slowenisch-österreischische Beziehungen im 20. Jahrhundert. Ljubljana: Oddelek za zgodovino Filozofske fakultete.

NEČAK, Dušan (2004) „'Nemci“ v Sloveniji 1938-1948.“ In: D. Nečak (et al.) (Hrsg), 349-402. 
PRINČIČ, Jože (1998) „Podržavljenje nemške imovine na slovenskem ozemlju po drugi svetovni vojni 1945-1955.“ In: D. Nečak (Hrsg.) (1998a), 245-270.

REPE, Božo (1998) „»Nemci« na Slovenskem po drugi svetovni vojni.“ In: D. Nečak (Hrsg.) (1998a), 145-172.

SUPPAN, Arnold (1988) „Zur Lage der Deutschen in Slowenien zwischen 1918 und 1938. Demographie - Recht - Gesellschafz - Politik.“ In: H. Rumpler/A. Suppan (Hrsg.), Geschichte der Deutschen im Bereich des heutigen Slowenien 1848-1941. Wien/München: R. Oldenbourg.

VOVKO, Andrej (1986) „Nemško manjšinsko šolstvo na Slovenskem v obdobju stare Jugoslavije." Zgodovinski časopis, 40, 3, 311-321.

WILDMANN, Georg/Hans SONNLEITNER/Karl WEBER (2000) Verbrechen an den Deutschen in Jugoslavien 1944-1948. Die Stationen eines Völkermords. München: Donauschwäbische Kulturstiftung.

ZAJŠEK, Boštjan (2011) Nemški evangeličani na Slovenskem med obema svetovnima vojnama. Kronika, 59, 91-106.

ZAVADLAV, Zdenko (1990) Iz dnevniških zapiskov mariborskega oznovca. Maribor: Založba za alternativno teorijo.

ZUPAN, Gojko/Mitja FERENC/Martin M. DOLINAR (1993) Cerkve na Kočevskem nekoč in danes/Die Gottscheer Kirchen einst und heute. Ljubljana: Župnija Kočevje. ZWITTER, Fran (1993) „Nemci na Slovenskem.“ Sodobnost, 6, 3, 483-497.

\section{Zusammenfassung}

\section{DAS SCHICKSAL DER DEUTSCHEN SPRACHMINDERHEIT IN SLOWENIEN}

Mit dem Zerfall der österreichisch-ungarischen Monarchie fand sich eine halbe Million Personen mit Deutsch als Muttersprache im neugegründeten jugoslawischen Staat wieder. Der kleinere Teil, rund 30.000, lebte im Jahr 1931 auf dem Gebiet des heutigen Slowenien, wo diese Menschen 2,5 \% der Bevölkerung darstellten. Sie waren in wirtschaftlicher und sozialer Hinsicht der stärkste und am besten organisierte Teil der deutschen Sprachminderheit. Obwohl der Umfang der Rechte, die die Deutschen im Vergleich zu den übrigen Sprachminderheiten genossen, am größten war, waren diese immer von der jeweiligen Politik der jugoslawischen Regierungen abhängig.

Nach dem Zweiten Weltkrieg nutzten die neuen jugoslawischen Behörden die Ethnozid-Besatzungspolitik des deutschen Staates und vor allem die Mitwirkung eines Teils der Angehörigen der deutschen Minderheit an dieser Politik für die endgültige Auseinandersetzung mit den Deutschen aus. Zunächst wurden sie in Konzentrationslager gebracht und danach aus dem Staat vertrieben, wobei ihr Vermögen konfisziert wurde. Einige Hundert wurden außergerichtlich liquidiert. Die Auseinandersetzung mit den Deutschen in der Nachkriegszeit war so heftig, dass in Slowenien nur noch ,verstreute Reste" der Minderheit übrigblieben. Auf der Grundlage der Volkszählungen wird belegt, dass die Anzahl der Deutschen und Österreicher bzw. der Personen mit Deutsch als Muttersprache niemals wieder an nicht einmal ein Zehntel der Anzahl vor 
dem Krieg heranreichte. Neben der kleinen Anzahl zählen zu den weiteren Merkmalen der deutschsprachigen Volksgruppe ihre territoriale Zerstreuung, ihr Nicht-Autochthonismus, ihre hohe Umzugsfrequenz und auch eine spezifische, vom slowenischen Durchschnitt abweichende Bevölkerungsstruktur.

Bei der letzten Volkszählung im Jahr 2002 wurden nur 963 Personen mit Deutsch als Muttersprache gezählt. Nach dem Kriterium der Nationalität gab es 181 Österreicher und 499 Deutsche. Nach den demokratischen Veränderungen im Jahr 1990 wurden mehrere deutsche Vereine gegründet. Die Erwartungen der Republik Österreich bezüglich der verfassungsrechtlichen Anerkennung der deutschen Minderheit in Slowenien regelten die Staaten im Jahr 2001 mit einem Kulturabkommen. Mit diesem Abkommen sind weder die deutschsprachige Gemeinschaft in Slowenien noch die Republik Österreich zufrieden, weil sie eine verfassungsrechtliche Anerkennung der deutschen Sprachminderheit in Slowenien und Rechte erwarten, die mit denen der italienischen und ungarischen Minderheit vergleichbar wären.

Schlüsselwörter: deutsche Minderheit, AVNOJ-Verordnungen, Gottscheer Deutsche, Vertreibung

\section{Abstract \\ THE FATE OF THE GERMAN-SPEAKING MINORITY IN SLOVENIA}

Half a million speakers of German found themselves living in the new Yugoslav state after the disintegration of the Austro-Hungarian Empire. A small portion of them (about 30,000 people or 2.5\% of the population) lived in present-day Slovenia in 1931. In economic and social terms, they represented the most powerful and organized part of the German minority. Although the German-speaking minority had more rights than any other minority, these were contingent upon Yugoslav governments' policy. After World War II, the new Yugoslav authorities made use of the German state's ethnocide and occupation, and particularly the participation of members of the German minority in it, to settle scores with the German population once and for all. They were interned in concentration camps and then expelled from the country with their belongings confiscated. Several hundred people were subject to extrajudicial killings. The post-war retributions were so severe that only scattered remnants of the minority remained in Slovenia. It can be gathered from the population censuses that the number of Germans and Austrians, i.e. people identifying German as their mother tongue, never even reached a tenth of the number recorded before the war. The minority is characterized by its small number, geographical dispersion, non-native status, migrations, and a demographic structure that diverges from the Slovene average. A mere 963 people identifying German as their mother tongue, 181 of whom identified as Austrian and 499 as German, were recorded in the 2002 population census. A number of German associations emerged after the democratic changes in 1990. The Republic of Austria's expectations that the German-speaking minority would achieve constitutional recognition in 
Slovenia were addressed by means of a cultural agreement between the states in 2001 . However, this does not satisfy the expectations of the German-speaking community in Slovenia or of the Republic of Austria. They call for the constitutional recognition of the German-speaking minority in Slovenia, as well as for rights comparable to those granted to the Italian and Hungarian community.

Keywords: German-speaking minority, AVNOJ decrees, the Gottschee Germans, expulsion

\section{Povzetek \\ USODA NEMŠKE MANJŠINE NA SLOVENSKEM}

$\mathrm{Z}$ razpadom avstro-ogrske monarhije se je $\mathrm{v}$ novi jugoslovanski državi znašlo pol milijona oseb z nemškim maternim jezikom. Manjši del, okoli 30.000, jih je leta 1931 prebival na ozemlju današnje Slovenije, kjer so predstavljali 2,5 \% prebivalstva. Bili so gospodarsko, socialno najmočnejši in najbolj organizirani del nemške manjšine. Čeprav so Nemci med manjšinami uživali še največ pravic, so bile te odvisne od vsakokratne politike jugoslovanskih vlad. Po 2 . svetovni vojni so nove jugoslovanske oblasti etnocidno okupacijsko politiko nemške države, predvsem pa sodelovanje dela pripadnikov nemške manjšine v njej, izkoristile za dokončen obračun z Nemci. Najprej so jih zaprli $\mathrm{v}$ koncentracijska taboriščih, zatem pa pregnali iz države in zaplenili njihovo premoženje. Nekaj sto so jih izvensodno likvidirali. Povojni obračun z Nemci je bil tako silovit, da so v Sloveniji ostali le še „raztreseni“ ostanki narodne manjšine. Popisi prebivalstva ugotavljajo, da število Nemcev in Avstrijcev oziroma oseb z nemškim maternim jezikom nikoli ni več doseglo niti desetine predvojnega števila. Poleg maloštevilnosti jih označuje še prostorska razpršenost, neavtohtonost, velike selitvene značilnosti in posebna strukturiranost, ki odstopa od slovenskega povprečja. V zadnjem popisu leta 2002 so našteli le 963 oseb z nemškim maternim jezikom, po narodni pripadnosti je bilo 181 Avstrijcev in 499 Nemcev. Po demokratičnih spremembah leta 1990 je nastalo več nemških društev. Pričakovanja Republike Avstrije po ustavno pravnem priznanju nemške manjšine v Sloveniji sta državi leta 2001 rešili s kulturnim sporazumom. Ta ne zadovoljuje nemško govoreče skupnosti v Sloveniji niti Republike Avstrije, ki pričakujeta ustavno priznanje nemške manjšine v Sloveniji in z italijansko in madžarsko narodno skupnostjo primerljive pravice.

Ključne besede: nemška manjšina, avnojski odloki, kočevski Nemci, izgon 